\title{
Distributed Load-Side Frequency Regulation for Power Systems
}

\author{
Congchong Zhang, Tao Liu, David J. Hill \\ Department of Electrical and Electronic Engineering \\ The University of Hong Kong \\ Hong Kong, China \\ Email: \{cczhang, taoliu, dhill\}@eee.hku.hk
}

\begin{abstract}
This paper studies frequency control of power systems by coordinating generation-side control and load-side control with nonlinear network-preserving models. A distributed consensus-based controller is designed for each bus in the transmission network. The total power imbalance of the system is discovered periodically by a distributed consensus mechanism, and then compensated by both generators and controllable loads accordingly. It is shown in simulation studies that the proposed method can achieve frequency regulation more effectively than the traditional automatic generation control (AGC) and reduce the needed system spinning reserve significantly. The impact of renewables on the system frequency under the designed control method is also discussed systematically in this paper.
\end{abstract}

Index Terms-Average consensus, distributed demand-side control, frequency control, optimal communication network.

\section{INTRODUCTION}

Traditionally, frequency control is implemented on the generation side by adjusting the mechanical power input of generators to follow demand, which consists of three layers working at different timescales [1]-[4]. The primary frequency control, also called droop control, operates on a timescale up to tens of seconds to rematch the supply with demand after disturbances and stabilize the frequency to a new equilibrium point in a decentralized way. But it cannot restore the frequency to its nominal value. The secondary frequency control, also known as the automatic generation control (AGC), operates on a timescale of 10 minutes to adjust the load reference base-points of selected generators in a centralized way [1]. It regulates the frequency to the nominal value and maintains the interchange power between control areas at scheduled values. The tertiary control, or economic dispatch, operates on a timescale of several minutes or more to schedule outputs of generators and interchange power between control areas.

However, due to the uncertainty and intermittency of renewable power, the traditional generation side frequency control may be inadequate to keep the system frequency at its nominal value [5]. The situation becomes even worse as the penetration

The work described in this paper was partially supported by a grant from the Research Grants Council of the Hong Kong Special Administrative Region under Theme-based Research Scheme through Project No. T23-701/14-N. of renewable power increases to higher levels, and a large extra quantity of spinning reserves will be required [6], whose costs are extremely high.

To solve this issue, efforts have been made from two different aspects. On one hand, new control methods proposed in [4], [7], [8] are adopted on the generation side to enhance or replace the traditional AGC. On the other hand, participation of the load side in frequency control has drawn a lot of attention because of its advantages such as instantaneous response, potentially lower costs and highly distributed availability throughout the whole network [9], [10]. For instance, load-side control has been adopted in [10], [11], to regulate the system frequency in which wind power fluctuation is the main disturbance to the system. Two types of load control methods have been designed for frequency regulation in [10]. Decentralized and distributed load-side control strategies have been adopted to the primary frequency control and secondary frequency control in [3], [12], respectively. In [13], an averageconsensus-based load shedding algorithm is used to maintain the supply-demand balance. Actual electricity loads such as aluminum smelter loads and refrigerators are used for frequency control in [11], [14], respectively, which shows the feasibility of load-side control in practical ways.

However, most of existing research works only consider frequency control either on the generation side or on load side instead of combining them together, which may not fulfill the need of future power systems with high penetration of renewables, particularly, when sensing and actuation communication is limited [15]. Since there may be millions or even billions of loads in the system, it may be impractical to adopt centralized control on the load side. Moreover, centralized control depends heavily on communication, which is vulnerable to communication failures [16]. In contrast, distributed control for the load side is more reasonable and feasible in which communication only exists between neighbors [12], [17]. But issues in the communication network will arise if distributed control is adopted on the load side, e.g., which buses should communicate with each other. Thus, the topology of the communication network of power system should be designed carefully to guarantee the robustness and efficiency of distributed control, which may have a different topology from that of the transmission network [18]. However, few 
research works have further investigate the impact of the topology of the communication network on frequency control.

This paper designs a distributed consensus-based controller for both controllable loads and generators to regulate the system frequency after disturbances. A distributed consensus mechanism is used for each controller to discover the average power imbalance of the system locally and periodically, which will be compensated by generators and controllable loads accordingly. Communication only exists between neighbors making the system more robust to communication failures. A hybrid algorithm combining the properties of small-world networks and the greedy heuristic is used to design the topology of the communication network in this paper.

The rest of this paper is organized as follows. In Section II the system model is introduced. In Section III the proposed distributed consensus-based controller is given. Section IV gives a hybrid algorithm for communication network design. Simulation results are presented and analyzed in Section V. The paper ends by conclusion in Section VI.

\section{SYSTEM MODEL}

In this paper, we make following assumptions:

1) The transmission network is connected in which transmission lines are lossless and characterized by reactances $x_{i j}=x_{j i}$.

2) The magnitude of the voltage of each bus in the grid $\left|V_{i}\right|$ is fixed such that active power flows between buses will not be affected by the voltage.

The network-preserving model for power systems proposed in [19] is adopted

$$
\begin{aligned}
\dot{\delta}_{i} & =\omega_{i}, i \in \mathcal{G} \\
M_{i} \dot{\omega}_{i} & =-D_{i} \omega_{i}+P_{i}^{m}-\sum_{j=1}^{N} b_{i j} \sin \left(\delta_{i}-\delta_{j}\right)-d_{i}, i \in \mathcal{G} \\
D_{i} \dot{\delta}_{i} & =u_{i}-\sum_{j=1}^{N} b_{i j} \sin \left(\delta_{i}-\delta_{j}\right)-d_{i}-P_{D_{i}}^{0}, i \in \mathcal{L}
\end{aligned}
$$

where $\mathcal{N}=\{1,2, \ldots, N\}=\mathcal{G} \cup \mathcal{L}$ is the index set of all buses where $\mathcal{G}$ and $\mathcal{L}$ are the index sets of generator buses and load buses, respectively. For $i \in \mathcal{G}, M_{i}, D_{i}, \delta_{i}, \omega_{i}$ and $P_{i}^{m}$ are the inertia constant, damping coefficient, phase angle, angular velocity and mechanical power input, respectively. For $i \in \mathcal{L}, D_{i}, u_{i}$ and $P_{D_{i}}^{0}$ are the frequency-dependent coefficient, controllable loads with capacity limits $\underline{u}_{i} \leq u_{i} \leq \bar{u}_{i}$ and uncontrollable loads, respectively. For $i \in \mathcal{N}, b_{i j}=\frac{\left|V_{i}\right|\left|V_{j}\right|}{x_{i j}}$, and $d_{i}$ is the power imbalance of each bus at the current operating point caused by the wind power fluctuation or an increase or decrease of load.

It should be noted that (1c) can also be used to describe inverter-based generators such as a wind farm or PV due to their lack of inertia [20]. For each inverter-based generator $i$ described by (1c), different from the load bus, $D_{i}$ represents the frequency dependence due to the inverter droop, and there is no uncontrollable loads $P_{D_{i}}^{0}$.
Remark 1: We only consider lossless transmission networks with aggregate loads which consist of different types of devices such as electric vehicles, air conditioning and electric springs [21] in this paper. It should be pointed out that the response of aggregate loads obtained can be used as a reference signal for the load devices in the sub-transmission network and distribution network. Thus, the problems of how to revise the proposed control method for the middle and low voltage networks which are lossy and how to use granular control to aggregate thousands or millions of low-voltage controllable loads to achieve the expected transmission-networklevel power supply across multiple time scales need to be considered in the future.

Remark 2: The disturbances considered in this paper are large disturbances, and hence it is not appropriate to use the linearized model for the problem considered. Stability of the system with the proposed control method is of great importance and needs to be studied in the future. Besides, the communication is usually implemented in a discrete way in practice, and thus the discrete-time distributed controller with discrete communication is more practical and should be adopted in the future.

\section{Distributed CONTROLlER DESIGN}

The consensus algorithm is an efficient mechanism to discover global information among a group of agents in a distributed way by using local communication. Thus, we adopt the consensus-based controller for both generators and controllable loads to discover the average power imbalance of the system periodically and then compensate it accordingly. The controller is given as follows

$$
\begin{aligned}
\dot{r}_{i}(t) & =\sum_{j=1}^{N} a_{i j}\left(r_{j}(t)-r_{i}(t)\right), t \in\left[t_{k}, t_{k}+T\right) \\
r_{i}\left(t^{+}\right) & =d_{i}(t), \quad t=t_{k}=k T, k=0,1,2, \ldots \\
u_{i}(t) & =u_{i}\left(t_{k-1}^{\prime}\right)+r_{i}^{a v g}\left(t_{k}\right)-\alpha_{i} \omega_{i}(t), t \in\left[t_{k}^{\prime}, t_{k+1}^{\prime}\right)
\end{aligned}
$$

where $r_{i}, \alpha_{i}$ and $T$ are the state of the designed controller, control gain and power imbalance sampling period, respectively. The matrix $A=\left(a_{i j}\right) \in \mathcal{R}^{N \times N}$ with $a_{i j}=a_{j i}>0$ if there is a communication connection between bus $i$ and bus $j$ and $a_{i i}=0$ is the adjacency matrix representing the topology of the communication network. In this paper, a hybrid algorithm based on the small-world network and the greedy heuristic is used to design the topology of the communication network (see the next section for details).

At each $t=t_{k}$, each controller samples the power imbalance of bus $i$ at $t=t_{k}$, i.e., $d_{i}(t)$ in (2b), according to which the state $r_{i}(t)$ is initialized, and starts to achieve consensus through communications with neighboring controllers to discover the average power imbalance of the system, i.e., $r_{i}^{a v g}\left(t_{k}\right)=\frac{1}{N} \sum_{j=1}^{N} d_{i}\left(t_{k}\right)$. Since it may take too long time to achieve the exact average power imbalance, it is necessary to define a terminating criterion. Thus, in this paper each 
controller will terminate the information discovery process as soon as the following inequality becomes valid

$$
\sum_{j=1}^{\mathcal{N}_{i}}\left|\left(r_{j}(t)-r_{i}(t)\right)\right|<\epsilon
$$

where $\epsilon>0$ is a constant that defines the precision requirement, and $\mathcal{N}_{i}=\left\{j \mid a_{i j}>0, j \in \mathcal{N}\right\}$ denotes the set of neighbors of controller $i$. The controller will send a consensus achievement notification signal to the control centre when condition (3) is satisfied. Once the control centre receives consensus achievement notification signals from all controllers, it will send a signal to each controller for control input update notification (communication delay is assumed to be negligible in this paper). Then each controller will update the term $r_{i}^{a v g}\left(t_{k}\right)$ in (2c) at $t=t_{k}^{\prime}$ (here we assume $t_{k}<t_{k}^{\prime}<t_{k}+T$ ) which remains unchanged until the average power imbalance of next period is discovered at $t=t_{k+1}^{\prime}$.

The term $\alpha_{i} \omega_{i}(t)$ in (2c) handles the instantaneous power imbalance during the period $\left[t_{k}^{\prime}, t_{k+1}^{\prime}\right)$, and serves as the primary frequency control. The term $r_{i}^{a v g}\left(t_{k}\right)$ compensates the power imbalance, and hence restores the frequency to the nominal value, which serves as the secondary frequency control.

The power imbalance sampling period $T$ should be considered carefully. On one hand, the smaller the $T$ is, the better the control performance could be expected as the real-time power imbalance of the system could be compensated in time. On the other hand, controllers will need time to achieve consensus which needs to be smaller than $T$, i.e. $t_{k}^{\prime}-t_{k}<T$. Thus, $T$ is set to 2 seconds in this paper since the execution of generationside control every 2 to 4 seconds results in good performance [1]. Meanwhile, it means that the average power imbalance of the system, i.e. $r_{i}^{a v g}\left(t_{k}\right)$ in (2c), need be achieved within 2 seconds.

\section{Communication Network Topology Design}

In the proposed control method, the average power imbalance of the system is supposed to be discovered within the time interval $\left[t_{k}, t_{k}+T\right)$ as soon as possible, otherwise the controller of each bus cannot update its control input, i.e., $u_{i}(t)$ in (2c) in time, which may lead to the frequency divergence. The convergence speed of state $r_{i}$ in (2a) depends on the topology of the communication network such that it will have big impact on the performance of the distributed consensusbased controller. Thus, we will design the topology of the communication network based on the graph theory.

Here, we consider the communication network as an undirected graph. For an undirected graph, its Laplacian can be described as $L=D-A$ where $D=\operatorname{diag}\left(d_{1}, \ldots d_{n}\right)$ is the degree matrix of the graph with elements $d_{i}=\sum_{j \neq i} a_{i j}$ and zero off-diagonal elements. The second smallest eigenvalue of $L$, i.e. $\lambda_{2}>0$ (if the network graph is connected), is called the algebraic connectivity of a graph, and is used to measure the performance of consensus algorithm [22]. In other words, the larger the algebraic connectivity $\lambda_{2}$ is, the faster the average power imbalance of the system $r_{i}^{a v g}\left(t_{k}\right)$ could be dicovered.
The topology of the communication network to be designed may be different from that of the transmission network [18]. This may give us more degrees of freedom to enlarge the algebraic connectivity $\lambda_{2}$ in order to improve the control performance. It has been shown in [23] that small-world networks have a significantly large $\lambda_{2}$ compared with regular networks with nearest neighboring topology. Thus, inspired by [18], [23] and [24], to design the topology of the communication network, a hybrid algorithm based on the small-world networks and greedy heuristic is used.

In this algorithm, each node is assumed to be initially connected with its $k$-nearest neighboring nodes and the network graph is denoted by $G_{\text {base }}$. Let $Q_{i}=\mathcal{N} \backslash\left(\mathcal{N}_{i} \cup\{i\}\right)$ denote the set of nodes that are not connected to the node $i$ initially. Then, each link $e=(i, j)$ of $G_{b a s e}$ is rewired with probability $p$ by replacing $(i, j)$ with $\left(i, j^{\prime}\right)$ uniformly at random with probability $q_{i}=1 /\left|Q_{i}\right|$ where $j \in Q_{i}$. A smallworld network is obtained after this operation and denoted by $G_{s m}$. Based on $G_{s m}, m$ new links are added one at a time. At each time, the link $e=(i, j)$ that has the largest value of $\left(v_{i}-v_{j}\right)^{2}$ where $v_{i}$ is the $i$ th entry of the eigenvector of $L$ corresponding to $\lambda_{2}$ is chosen to be added. The network graph after $m$ links being added is denoted by $G_{h}$, which represents the topology of the communication network. The procedure of this algorithm could be illustrated briefly as follows :

1) A regular network graph $G_{\text {base }}$ where each node is connected to $k$ nearest-neighboring nodes is given.

2) To get the small-world network $G_{s m}$, each link $e=(i, j)$ of $G_{b a s e}$ is replaced with a new link $\left(i, j^{\prime}\right)$ with probability $p$.

3) Starting from $G_{s m}, m$ new links $(i, j)$ with largest $\left(v_{i}-\right.$ $\left.v_{j}\right)^{2}$ are added one at a time to formulate the topology of the communication network $G_{h}$.

Remark 3: It should be pointed out that the time that controllers spend in achieving consensus needs to be smaller than $T$. The original physical network usually has sparse links between nodes which may lead to a slow convergence speed of the state $r_{i}$, and hence will influence the performance of controllable loads since $r_{i}^{a v g}$ in (2c) will not be updated until the consensus is achieved. The proposed hybrid algorithm makes a trade-off between the convergence speed and the total number of links in the communication network, and results in a larger algebraic connectivity $\lambda_{2}$ which can improve the performance of load-side control. Other algorithms proposed in the related literature (e.g. [13]) can also be used to design the communication network.

Remark 4: The average power imbalance obtained by each controller will not be influenced by communication failures if the communication network is still connected. Thus, the proposed control method is more robust to communication failures than the centralized control method from this point of view. However, communication failures will influence the consensus convergence speed which may in turn degrade the control performance of the entire system. Thus, the robustness of the communication network to communication failures is of great importance and hence should be studied in the future. 
Remark 5: The issue of node or line outage during the global information discovery process should be dealt with carefully since it will influence the final value of the consensus and consequently the performance of the proposed control method. Some basic ideas such as the one proposed in [13] can be adopted to overcome this issue.

\section{Simulation Results}

In this section, the proposed control method is tested with the IEEE 9-bus system and the IEEE 39-bus system, respectively. The impact of wind power fluctuation to the system frequency, the response of controllable loads and the mechanical power input of generators in different scenarios will be demonstrated in detail in this section.

The nominal value of frequency and per unit of active power are $50 \mathrm{~Hz}$ and $100 \mathrm{MVA}$ in all simulation studies, respectively. The system frequency mentioned in this section is calculated as $f=\frac{\sum_{i=1}^{N} D_{i} \omega_{i}}{2 \pi \sum_{i=1}^{N} D_{i}}$. We assume each generator participating in frequency control has the reheat steam turbine, the parameters of which can be acquired from [1]. At each load bus, we assume $10 \%$ of the load is controllable. It should be noticed that in this section we use the following index $\mathcal{P}$ to define the percentage of wind power penetration

$$
\mathcal{P}=\frac{\sum_{i=1}^{N_{w p}} P_{i}^{w p}}{\sum_{i=1}^{N_{w p}} P_{i}^{w p}+\sum_{i=1}^{N_{c g}} P_{i}^{c g}} \times 100 \%
$$

where $P_{i}^{w p}$ and $P_{i}^{c g}$ are the power output of wind farm $i$ and the mechanical power input of conventional generator $i$ within the same time interval, respectively. $N_{w p}$ and $N_{c g}$ are the number of wind farms and conventional generators in the system, respectively. The wind power data used in the simulation is acquired from [25]. In order to achieve different $\mathcal{P}$ in each case, we use the same wind power data with different proportion in the total generation.

\section{A. IEEE 9-bus system}

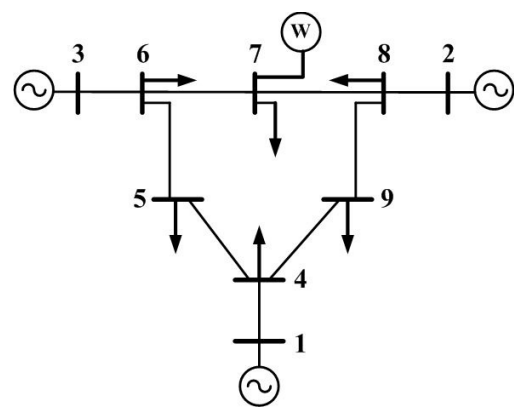

Figure 1. The IEEE 9-bus system

In this subsection, the IEEE 9-bus system consisting of 3 generators and 6 loads (Fig. 1) is used to test the proposed control method. Cases with a contingency of generation outage and wind power fluctuation are simulated to compare the frequency regulation performance of the proposed control method with the traditional AGC.
Parameters of the system can be found in [1]. $30 \mathrm{MW}$ active power loads are assumed to be added to bus 4,6 and 8 , respectively. A wind farm with $\mathcal{P}=32.5 \%$ is assumed to be located at bus 7 . The control gain $\alpha_{i}$ for each controller is uniformly set to 20 for simplicity, which is the same as the droop constant of the generator. The precision requirement of consensus is taken as $\epsilon=10^{-6}$.

1) The topology of the communication network is designed based on the algorithm discussed in Section IV. In the basic graph $G_{\text {base }}$, each bus is assumed to be connected with 2 nearest buses. To form the small-world network, each link $e=(i, j)$ of $G_{\text {base }}$ is replaced with a new link $\left(i, j^{\prime}\right)$ with a probability $p=0.5$. Then, $m=3$ new links are added to the network one at a time to achieve the final network graph $G_{h}$. The algebraic connectivity $\lambda_{2}$ with different network topologies is shown in Tab. I. The algebraic connectivity $\lambda_{2}$ of $G_{h}$ is nearly 6 times larger than the one has the same topology as the transmission network.

TABLE I. ThE ALGEBRAIC CONNECTIVITY $\lambda_{2}$ WITH DIFFERENT COMMUNICATION NETWORK TOPOLOGIES

\begin{tabular}{|c|c|c|}
\hline Network topology & $\lambda_{2}$ & Links number \\
\hline Transmission network & 0.4679 & 9 \\
\hline Nearest neighbour $(\mathrm{k}=2)$ & 2.1206 & 18 \\
\hline Hybrid algorithm & 2.7460 & 21 \\
\hline
\end{tabular}

2) Fig. 2 shows the system frequency, the response of controllable loads and the total mechanical power input of generators under the proposed control method and the traditional AGC, respectively, with a contingency of 0.1 p.u. generation outage at bus 3 at $t=150 \mathrm{~s}$. Controllable loads are not activated before the contingency occurs and act immediately when the outage occurs at $t=150 \mathrm{~s}$. As a result, we can observe in Fig. 2 that both the frequency overshoot and settling time of the proposed control method are significantly decreased compared with AGC. Moreover, the total mechanical power input of generators of the proposed control method has been effectively reduced 0.089 p.u. compared with AGC, which shows the proposed control method can save spinning reserves significantly.

3) A 30-minute window of the wind power with major fluctuation is shown in Fig. 3(b). We can observe in Fig. 3(a) that both the proposed control method and the traditional AGC result in satisfactory frequencies within the Normal Operating Frequency Band $([49.85,50.15] \mathrm{Hz})$ [26], but the proposed control method has a better frequency response $\left(f_{C L}\right)$ than AGC $\left(f_{A G C}\right)$. For instance, at $t$ around $1200 \mathrm{~s}, f_{A G C}$ drops from $50.02 \mathrm{~Hz}$ to $49.91 \mathrm{~Hz}$ whereas $f_{C L}$ drops from 50.00 $\mathrm{Hz}$ to $49.99 \mathrm{~Hz}$ as the wind farm power output decreases from 1.45 p.u. to 0.62 p.u..

Remark 6: It should be pointed out that in some research works, (e.g. [10]), the band [49.90,50.10] $\mathrm{Hz}$ is used as the Normal Operating Frequency Band. In such a situation, the proposed control method can still fulfill the requirement, but needs to adjust the contribution of controllable loads properly. 

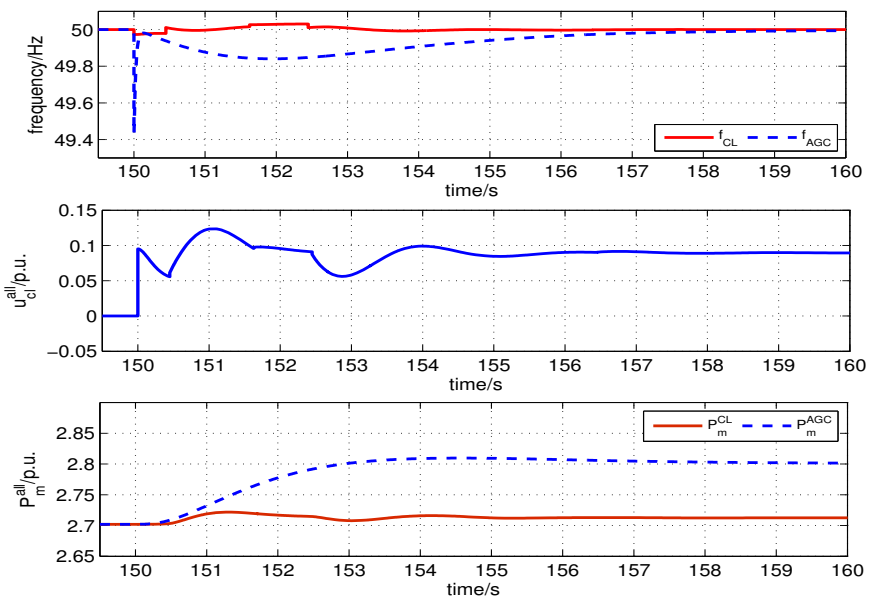

Figure 2. The system frequency (top), the aggregated response of controllable loads (middle) and the total mechanical power input of generators (bottom).

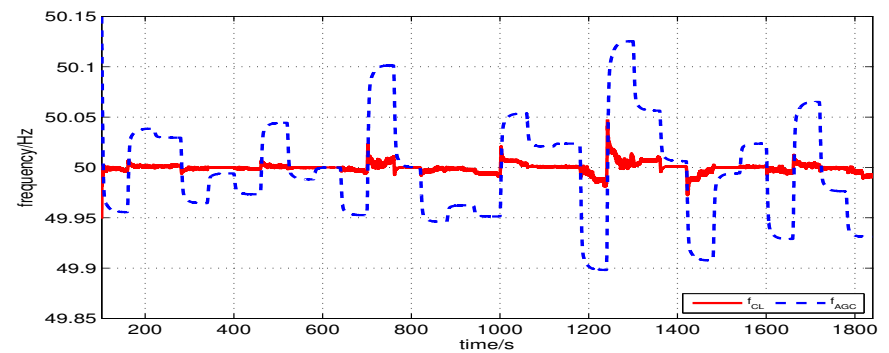

(a)
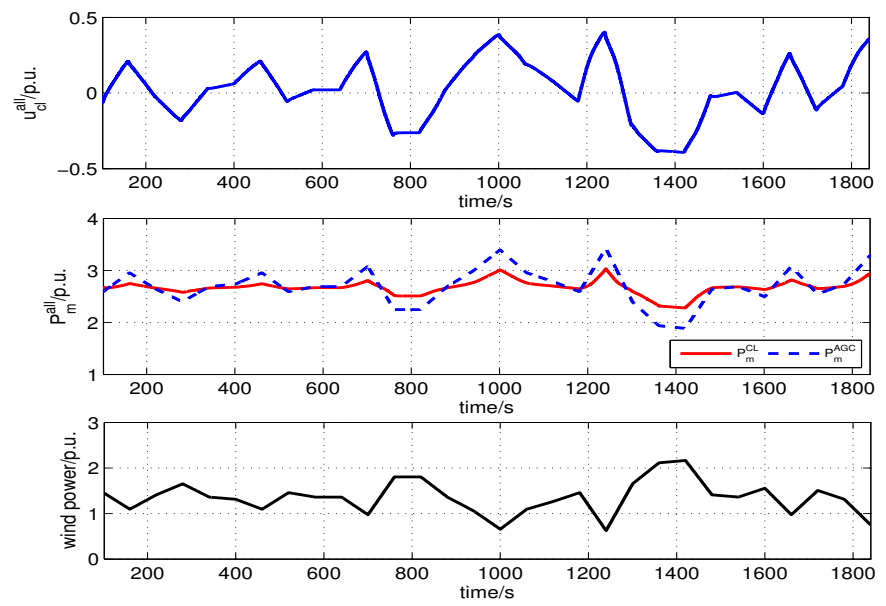

(b)

Figure 3. (a) The system frequency; (b) the response of aggregated controllable loads (top), the total mechanical power input of generators (middle) and active power output of wind farm (bottom).

\section{B. IEEE 39-bus system}

The more complex and widely used IEEE 39-bus system (Fig. 4) is used to test the proposed control method. Cases with different $\mathcal{P}$ and with different load capacity limits are simulated in this subsection.

Parameters of the test system are taken from [27] and the total system loads are $6254 \mathrm{MW}$. A wind farm is assumed to be located at bus 6 . The control gain $\alpha_{i}$ and precision requirement

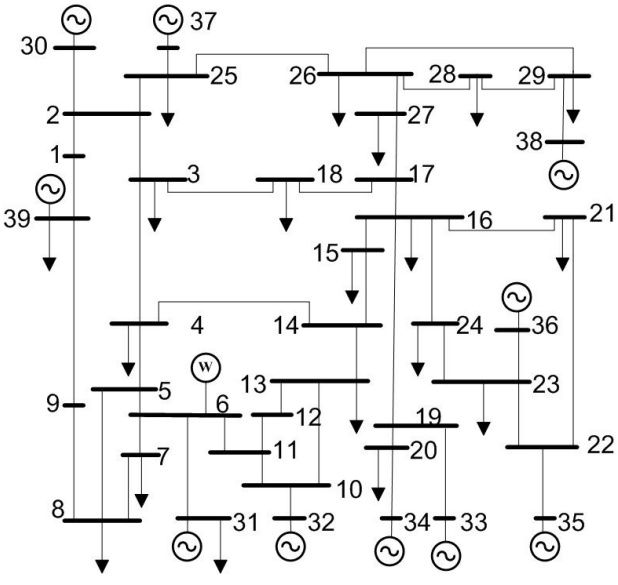

Figure 4. The IEEE 39-bus system

of consensus $\epsilon$ for each controller are uniformly set to 10 and $10^{-4}$, respectively.

1) The hybrid algorithm is also adopted in this test system such that in the basic graph $G_{b a s e}$, each bus is assumed to be connected with 3 nearest buses. The probability $p$ of each link being rewired is 0.5 and $m=10$ new links are added to the network one at a time to achieve the $G_{h}$. The algebraic connectivity $\lambda_{2}$ with different network topologies is shown in Tab. II. The algebraic connectivity $\lambda_{2}$ of $G_{h}$ is nearly 40 times larger than the one has the same topology as the transmission network. Thus, the proposed hybrid algorithm clearly increases $\lambda_{2}$ significantly, which leads to a fast convergence speed for consensus.

TABLE II. THE ALGEBRAIC CONNECTIVITY $\lambda_{2}$ WITH DIFFERENT COMMUNICATION NETWORK TOPOLOGIES

\begin{tabular}{|c|c|c|}
\hline Network topology & $\lambda_{2}$ & Links number \\
\hline Transmission network & 0.0762 & 46 \\
\hline Nearest neighbour $(\mathrm{k}=3)$ & 0.3579 & 117 \\
\hline Hybrid algorithm & 2.8418 & 127 \\
\hline
\end{tabular}

2) Fig. 5(a) shows the frequency response under the traditional AGC $\left(f_{A G C}\right)$ and the proposed control method $\left(f_{C L}\right)$ with $\mathcal{P}=40 \%$, respectively. It is obvious that $f_{A G C}$ exceeds the band $[49.85,50.15] \mathrm{Hz}$ when a big wind power fluctuation occurs. For instance, during $[700,750]$ s, $f_{A G C}$ drops from 50 $\mathrm{Hz}$ to $49.77 \mathrm{~Hz}$ as the power output of wind farm decreases from 25.2 p.u. to 18.0 p.u.. In contrast, $f_{C L}$ is closely around $50 \mathrm{~Hz}$ during the same interval, and within $[49.85,50.15] \mathrm{Hz}$ all the time. The magnitude of system frequency deviation of the proposed control method is much smaller than AGC because of the instantaneous response and highly distributed availability characteristics of controllable loads.

3) To discover the maximum allowable penetration of wind power that $10 \%$ controllable loads could handle, cases with different $\mathcal{P}$ with a $5 \%$ step increase from $50-65 \%$ shown in Fig. 6(a) are studied.

Fig. 6(b) gives system frequencies with different $\mathcal{P}$ from $50-65 \%$, respectively. It can be observed that the frequency 


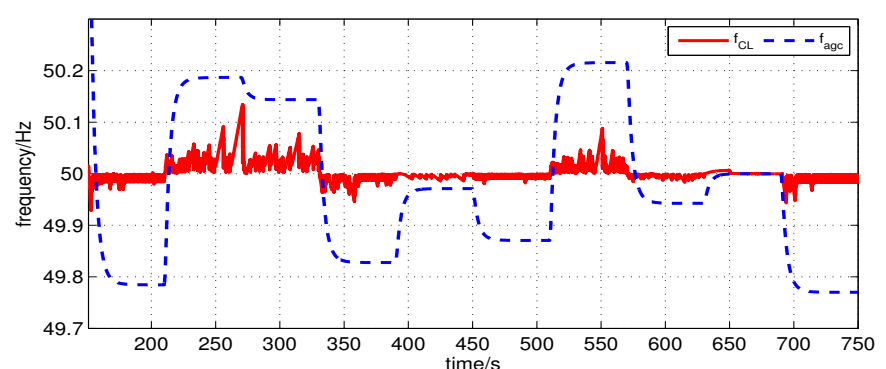

(a)
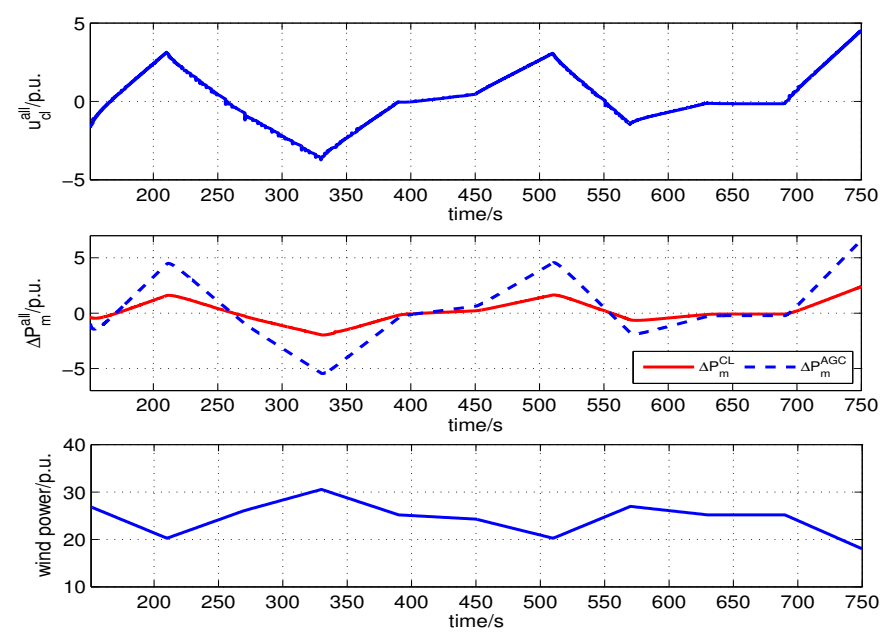

(b)

Figure 5. (a) The system frequency ( $f_{A G C}$ and $f_{C L}$, respectively); (b) the total adjustment of mechanical power input of generators $\left(\Delta P_{m}^{a l l}\right)$, the aggregated response of controllable loads $\left(u_{c l}^{a l l}\right)$ and active power output of wind farm, respectively.

deviation becomes larger when $\mathcal{P}$ increases to higher levels. Cases with different $\mathcal{P}$ except the one with $\mathcal{P}=65 \%$ all have satisfactory frequencies within $[49.85,50.15] \mathrm{Hz}$. Fig. 6(c) shows the aggregated response of controllable loads $\left(u_{c l}^{\text {all }}\right)$ and total mechanical power input of generators $\left(P_{m}^{a l l}\right)$, respectively. We observe that $u_{c l}^{a l l}$ increases proportionally due to the increase of wind power fluctuation as $\mathcal{P}$ increases to higher levels, which in all cases saves $P_{m}^{\text {all }}$ effectively. However, $u_{c l}^{\text {all }}$ with $\mathcal{P}=65 \%$ are almost the same as those with $\mathcal{P}=60 \%$ due to the saturation of controllable loads. As a result, the frequency with $\mathcal{P}=65 \%$ exceeds the band $[49.85,50.15] \mathrm{Hz}$ several times such as at $t=350 \mathrm{~s}$. However, we can still draw the conclusion that, in this situation, the penetration of wind power could increase at least $20 \%$ under the proposed control method with $10 \%$ load capacity limits compared with the traditional AGC which exceeds the band $[49.85,50.15] \mathrm{Hz}$ when $\mathcal{P}=40 \%$.

(4) We can observe in Fig. 7(b) that the system frequency is within $[49.85,50.15] \mathrm{Hz}$ all the time when load capacity limits are enlarged to $20 \%$. This result can be well explained by comparing responses of controllable loads shown in Fig. 7(b) with those shown in Fig. 7(a). It can be observed that controllable loads at both buses with $10 \%$ capacity limits saturate several

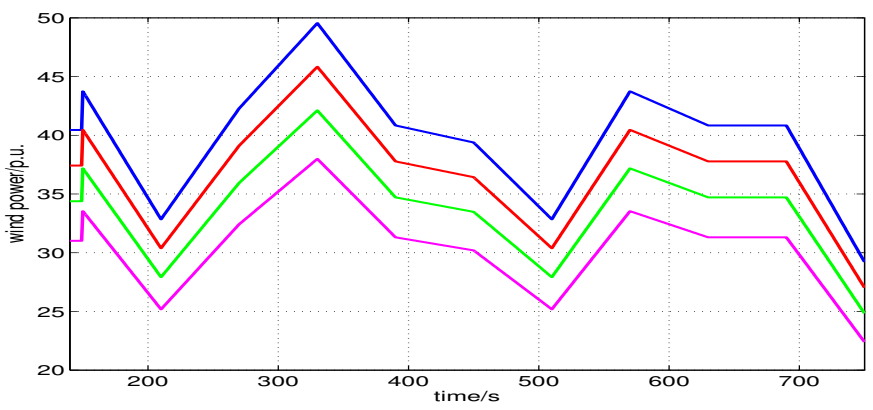

(a)
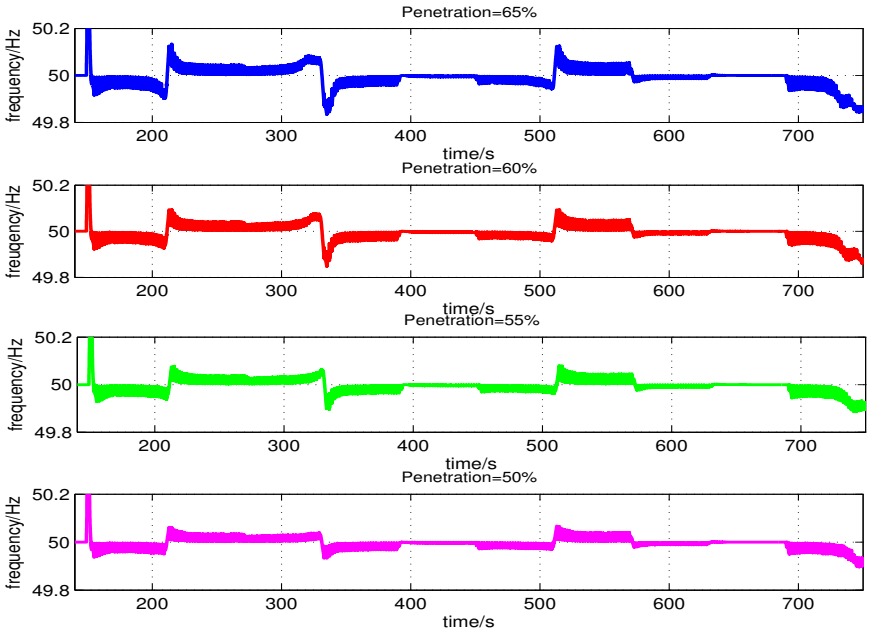

(b)
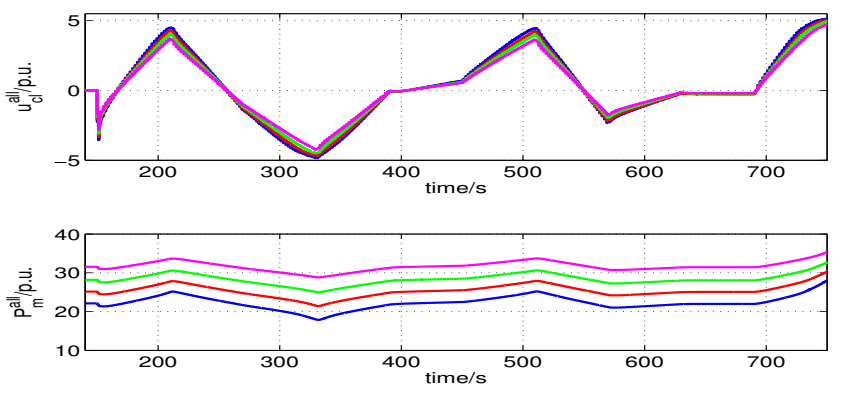

$65 \%$ penetration $-60 \%$ penetration $-55 \%$ penetration $-50 \%$ penetration

(c)

Figure 6. (a) Active power output of wind farm with different penetration; (b) system frequencies with different wind power penetration; (c) the aggregated response of controllable loads ( $u_{c l}^{a l l}$ in the top) and the total mechanical power input of generators $\left(P_{m}^{a l l}\right.$ in the bottom) with different wind power penetration.

times such as at $t=350 \mathrm{~s}$, at which the system frequency exceeds the band $[49.85,50.15] \mathrm{Hz}$. In contrast, controllable loads with $20 \%$ capacity limits still work very well when big wind power fluctuation occurs. Therefore, increasing load capacity limits may lead to better control performance when $\mathcal{P}$ increases to higher levels. However, issues such as how to achieve not only fully responsive to frequency control but also non-disruptive to end-use performance will arise. Some basic idea has been proposed in [28], but there are still a lot of problems that deserve attention in further study. 

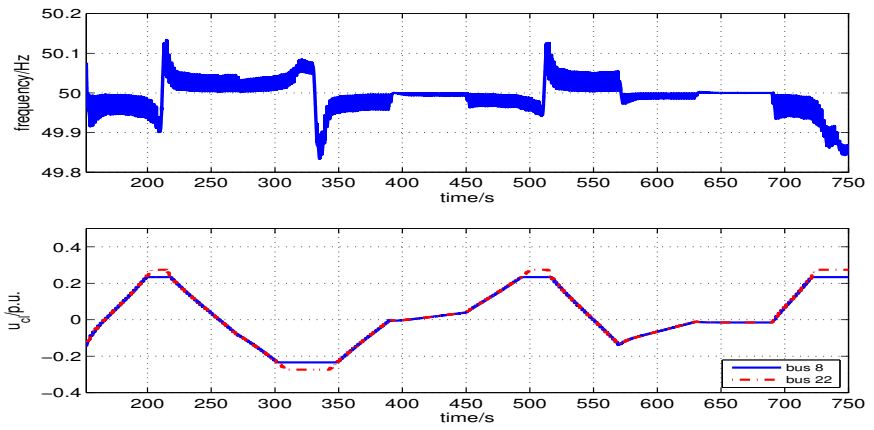

(a)
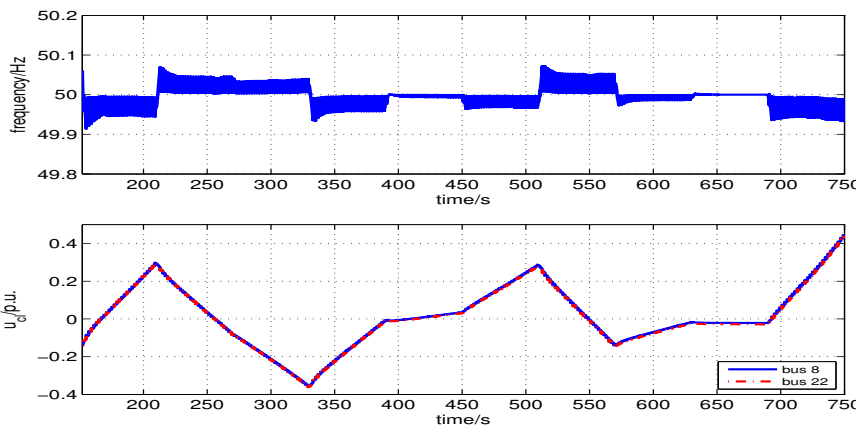

(b)

Figure 7. (a) The system frequency (top) and responses of controllable loads at bus 8 and 22 (bottom) with with $10 \%$ load capacity limits; (b) the system frequency (top) and responses of controllable loads at bus 8 and 22 (bottom) with with $20 \%$ load capacity limits.

\section{CONCLUSION}

A distributed consensus-based control method has been proposed for both controllable loads and generators to restore the system frequency after disturbances. The consensus mechanism has been adopted to discover the average power imbalance of system periodically, which serves an input of each controller to compensate. Besides, a hybrid algorithm has been used to design the topology of the communication network to improve the performance of power imbalance discovery.

Different scenarios have been simulated in the IEEE 9-bus system and 39-bus system to investigate the performance of the proposed method. Simulation results prove that the proposed control method has significant better frequency regulation performances than the traditional AGC whilst participation of controllable loads effectively reduces the needed spinning reserves. The maximum allowable wind power penetration with fixed percentage of controllable loads has also been investigated for IEEE 39-bus system, which increases at least $20 \%$ of wind power penetration comparing with AGC.

\section{REFERENCES}

[1] Kundur, Power system stability and control. McGraw-hill New York, 1994, vol. 7.

[2] A. R. Bergen and V. Vittal, Power systems analysis, 2nd ed. PreenticeHall, 2000.

[3] C. Zhao, U. Topcu, N. Li, and S. Low, "Design and stability of load-side primary frequency control in power systems," Automatic Control, IEEE Transactions on, vol. 59, no. 5, pp. 1177-1189, 2014.
[4] C. Wu, S. Kar, and G. Hug, "Enhanced secondary frequency control via distributed peer-to-peer communication," arXiv:1403.1868, 2014.

[5] R. Piwko, D. Osborn, R. Gramlich, G. Jordan, D. Hawkins, and K. Porter, "Wind energy delivery issues [transmission planning and competitive electricity market operation]," Power and Energy Magazine, IEEE, vol. 3, no. 6, pp. 47-56, Nov 2005.

[6] G. Strbac et al., "Quantifying the system costs of additional renewables in 2020," DTI report, 2002.

[7] M. Andreasson, D. V. Dimarogonas, H. Sandberg, and K. H. Johansson, "Distributed PI-control with applications to power systems frequency controlpi-control with applications to power systems frequency control," in American Control Conference, 2014, pp. 3183-3188.

[8] T. Mohamed, H. Bevrani, A. Hassan, and T. Hiyama, "Decentralized model predictive based load frequency control in an interconnected power system," Energy Conversion and Management, vol. 52, no. 2, pp. 1208-1214, 2011.

[9] D. S. Callaway and I. Hiskens, "Achieving controllability of electric loads," Proceedings of the IEEE, vol. 99, no. 1, pp. 184-199, 2011.

[10] Z. Xu, J. Østergaard, and M. Togeby, "Demand as frequency controlled reserve," Power Systems, IEEE Transactions on, vol. 26, no. 3, pp. 10621071, 2011.

[11] H. Jiang, J. Lin, Y. Song, and D. Hill, "MPC-based frequency control with demand-side participation: A case study in an isolated windaluminum power system," Power Systems, IEEE Transactions on, vol. 30, no. 6, pp. 3327-3337, Nov 2015.

[12] C. Zhao, E. Mallada, and F. Dorfler, "Distributed frequency control for stability and economic dispatch in power networks," in American Control Conference, 2015, pp. 2359-2364.

[13] Y. Xu, W. Liu, and J. Gong, "Stable multi-agent-based load shedding algorithm for power systems," Power Systems, IEEE Transactions on, vol. 26, no. 4, pp. 2006-2014, 2011.

[14] J. Short, D. G. Infield, and L. L. Freris, "Stabilization of grid frequency through dynamic demand control," Power Systems, IEEE Transactions on, vol. 22, no. 3, pp. 1284-1293, 2007.

[15] D. M. Prett and M. Morari, The shell process control workshop. Elsevier, 2013.

[16] C.-T. Lee, R.-P. Jiang, and P.-T. Cheng, "A grid synchronization method for droop-controlled distributed energy resource converters," Industry Applications, IEEE Transactions on, vol. 49, no. 2, pp. 954-962, 2013.

[17] J. W. Simpson-Porco, F. Dörfler, and F. Bullo, "Synchronization and power sharing for droop-controlled inverters in islanded microgrids," Automatica, vol. 49, no. 9, pp. 2603-2611, 2013.

[18] T. Liu, D. J. Hill, and J. Zhao, "Synchronization of dynamical networks by network control," Automatic Control, IEEE Transactions on, vol. 57, no. 6, pp. 1574-1580, 2012.

[19] A. R. Bergen and D. J. Hill, "A structure preserving model for power system stability analysis," Power Apparatus and Systems, IEEE Transactions on, no. 1, pp. 25-35, 1981.

[20] N. Ainsworth and S. Grijalva, "A structure-preserving model and sufficient condition for frequency synchronization of lossless droop inverterbased ac networks," Power Systems, IEEE Transactions on, vol. 28, no. 4, pp. 4310-4319, 2013.

[21] S. Y. Hui, C. K. Lee, and F. F. Wu, "Electric springs-a new smart grid technology," Smart Grid, IEEE Transactions on, vol. 3, no. 3, pp. 1552 1561, 2012.

[22] R. Olfati-Saber, A. Fax, and R. M. Murray, "Consensus and cooperation in networked multi-agent systems," Proceedings of the IEEE, vol. 95, no. 1, pp. 215-233, 2007.

[23] R. Olfati-Saber, "Ultrafast consensus in small-world networks," in American Control Conference, 2005, pp. 2371-2378.

[24] A. Ghosh and S. Boyd, "Growing well-connected graphs," in Decision and Control, IEEE Conference on, 2006, pp. 6605-6611.

[25] Y. Zheng, Z. Y. Dong, F. J. Luo, K. Meng, J. Qiu, and K. P. Wong, "Optimal allocation of energy storage system for risk mitigation of discos with high renewable penetrations," Power Systems, IEEE Transactions on, vol. 29, no. 1, pp. 212-220, Jan 2014.

[26] AEMO, "Power system frequency and time error monitoring," May 2013.

[27] J. Chow and G. Rogers, "Power system toolbox version 3.0, ontario: cherry tree scientific software, 2008."

[28] T. Liu, D. J. Hill, and C. Zhang, "Non-disruptive load-side control for frequency regulation in power systems," accepted by Smart Grid, IEEE Transactions on, 2016. 